\title{
Sharing \& Cooperativism: Designing For Economies
}

\author{
Özge Subasi \\ ozsubasi@ku.edu.tr \\ Department of Media and Visual Arts, \\ Koç University \\ Istanbul, Turkey
}

\author{
Anton Fedosov \\ antonf@ifi.uzh.ch \\ University of Zurich \\ Zurich, Switzerland
}

\author{
Oliver Bates \\ o.bates@lancaster.ac.uk \\ Lancaster University \\ Lancaster, UK
}

\author{
Airi Lampinen \\ airi@dsv.su.se \\ Stockholm University \\ Stockholm, Sweden
}

\author{
Ann Light \\ Ann.Light@sussex.ac.uk \\ University of Sussex \\ Brighton, UK
}

\begin{abstract}
Recent work on sharing and cooperativism has helped widen our understanding of the emerging systems for exchanges, interactions, and relationships beyond mainstream economic models, in particular through studying local cooperatives and their sharing practices across various domains. These efforts also indicate that design has the potential to shape our engagements with the global political economy. However, so far, there are few design resources tailored for exploring and further developing design insights from empirical and conceptual research on sharing and cooperativism. Therefore, we invite the community to discuss the role of design in relation to economies of sharing and cooperativism. In this workshop, we will gather a diverse group of scholars, designers, and activists to think together how designs for sustainable economies can be created and circulated across cooperatives and platforms, with the aim to springboard social and economic aspects of sharing cultures.
\end{abstract}

\section{CCS CONCEPTS}

- Human-centered computing $\rightarrow$ Human computer interaction (HCI).

\section{KEYWORDS}

cooperatives, collaborative economy, sharing, design, economic exchange

\section{ACM Reference Format:}

Özge Subasi, Anton Fedosov, Oliver Bates, Airi Lampinen, and Ann Light. 2020. Sharing \& Cooperativism: Designing For Economies. In Proceedings of the 11th Nordic Conference on Human-Computer Interaction: Shaping Experiences, Shaping Society (NordiCHI '20), October 25-29, 2020, Tallinn, Estonia. ACM, New York, NY, USA, 3 pages. https://doi.org/10.1145/3419249.3420094

\section{INTRODUCTION}

A recent turn in design [10] sets the expectation that designers take responsibility for bringing the political economy to designers'

Permission to make digital or hard copies of part or all of this work for personal or classroom use is granted without fee provided that copies are not made or distributed for profit or commercial advantage and that copies bear this notice and the full citation on the first page. Copyrights for third-party components of this work must be honored.

For all other uses, contact the owner/author(s).

NordiCHI '20, October 25-29, 2020, Tallinn, Estonia

(C) 2020 Copyright held by the owner/author(s).

ACM ISBN 978-1-4503-7579-5/20/10.

https://doi.org/10.1145/3419249.3420094 attention by asking who will benefit, and how to take measures to address the problems of ecology and inequality caused by profligate development. As an example, a small body of work on sharing and cooperativism within HCI shows the potential for new systems, platforms, and ecologies to harness social and ecological sustainability, as well as trust in platforms and communities [1, 4, 5, 7, 9]. These can be related to design decisions, and to the importance of designers taking a stance and shouldering responsibility [10]. However, despite the growing scholarship on cooperativism, the relation of design and economics $[6,8,10]$ is rarely addressed. Our aim is to foster such a discussion through a full day design workshop focused on the relationship between economics and design in the context of sharing and cooperativism.

In this workshop, we will engage with sharing as a collaborative economic strategy for managing resources by borrowing, lending, or collectively owning, acting, and using [9]. By placing alternative economic models and related design propositions to the center [8], we aim to explore three central aspects of sharing and cooperativism: (1) Local and relational assets of sharing; (2) Global principles of sharing cultures; and (3) Designing beyond markets. These themes can help us widen our design perspectives on the economics of sharing and cooperativism. In particular, we will look for common threads as to how designers can contribute to devising mechanisms for better allocation and use of underutilized resources (e.g., equipment, fertile land) and skills (e.g., freelance labor).

\section{WORKSHOP GOALS AND LEARNING OUTCOMES}

The primary goals of this workshop are to:

- Identify characteristics of design for sharing and cooperativism that can be used to inform and challenge current understandings of consumerism, assessing the potential for change.

- Critically reflect on how economic relations enable or limit sharing and cooperativism and how sharing cultures evolve over time and space.

- Collect theories and methodologies for motivating design to work on disrupting current limiting mechanisms. Synthesize a design taxonomy "for alternative economies" to springboard cooperativism in future HCI scholarship and design practice. 
- Gather a diverse community of scholars, designers, and sharing and cooperativism activists to foster future collaborations and to extend the current repository of projects (from previous workshops and gatherings at ACM conferences) for the HCI audience globally.

The expected outcomes for the participants are twofold: (1) designers and practitioners who aim to further examine design features concerning the economics of sharing and cooperativism will be able to turn to the design taxonomy with a view of operationalizing it in relation to their projects; (2) HCI researchers will be able to navigate the emergent links between the design and economics of sharing and cooperativism to better articulate their research objectives, contributing to a broader research agenda.

\section{A SCHEDULED OVERVIEW OF THE PLANNED ACTIVITIES}

We propose a one-day workshop with up to 20 participants from academia, industry, sharing associations, and local co-ops. The overall workshop structure will be roughly as follows (remote attendance possible):

09:00-10:30 Opening and Presentations (90 min) The organizers will kick-off the workshop with a brief presentation of the agenda, goals, and the format. They will then moderate a short round of lightning talks, providing each participant an opportunity to introduce themselves, their research interests, and thoughts stemming from their position paper.

\section{Coffee break (15 min)}

10:45-11:15 Introducing and Exploring Workshop Themes (30 min) The organizers will introduce themes that the workshop will cover: Local relational assets, Global sharing cultures, and Design beyond markets. Participants will contribute to the discussion by revisiting relevant research, case studies, and personal experiences of participants, suggesting any outstanding ethical, methodological and practical challenges, as well as asking open-ended questions to critically discuss the themes. We will facilitate the activity by splitting participants randomly into break-out groups and then bringing the groups back to report key ideas with the whole workshop. This phase will generate categories and sets of questions for the subsequent activity.

11:15-12:15 Analyzing Existing Platforms (60 min) Participants will be split into smaller groups (4-5 people) based on research interest and prior experience in each thematic area. In this session, participants will review and discuss existing platform co-ops/local initiatives (pre-selected by workshop organizers) in our three thematic categories. The organizers will offer additional topical areas (e.g., transport, housing, maker spaces, job sharing, skill-building opportunities) and will employ a set of tools (e.g., the Sharing Economy Design Cards [2] and the Platform Co-op Development Kit) to facilitate design-oriented discussions. We will make these toolkits available to the participants on our website prior to the beginning of the workshop such that they can get familiar with them in advance.

\section{Lunch (60 min)}

13:15-14:30 Creating a New Platform (75 min) The groups will continue working together, now with the task of conceiving an imaginary platform (or a value-added feature of an existing platform), defining possible users, success criteria, and the ecosystem (e.g., local relational assets). The goal is to collect distinctive design features that can be shared across various contexts and geographies.

14:45-15:30 Pecha Kucha Presentations (45 min) Each group will introduce the imaginary platform and the defined assets in the form of a Pecha Kucha presentation, connecting back to the outcomes of the theme discussion.

\section{Coffee break (15 min)}

15:30-17:00 Synthesis and Next Steps $(90$ min) The workshop will conclude with a group discussion reviewing what has been achieved, looking back at the initial design features selected prior to the discussions. Our goal is to elicit an outline (in the form of a design taxonomy) for future scholars and designers who aim to further examine design features in relation to the economics of sharing and cooperativism. This outline will form a backbone of a road-map for future scholarship and practice. Ultimately, we outline steps for further collaboration and networking.

\section{THE CALL FOR WORKSHOP PARTICIPATION}

The NordiCHI 2020 workshop "Sharing \& Cooperativism: Designing for Economies" aims to bring together practitioners and scholars working at the intersection of sharing, cooperativism, and design.

By turning to sharing and cooperativism, we imagine building systems in relation to the wider social and political structures surrounding these concepts. Yet, in sharing and cooperativism, less emphasis is given to the possibilities that design can bring to the emerging interactions at the intersections of social, cultural, and economic issues. A progressive approach is needed to uncover the relationship of sharing and cooperativism to new design paradigms that allows us to move towards an alternative understanding of economies through design. Our aim is to ground this discussion through a full day design workshop that is well-aligned with the historical, political, and economic infrastructures in order to shape the relationship of design and economics in the context of sharing and cooperativism.

We call for participants from diverse backgrounds to contribute to a critical discussion at the intersection of design, sharing, cooperativism, and economics on the workshop's thematic areas: (i) Local relational assets in sharing; (ii) Global sharing cultures, and (iii) Design beyond markets for sharing and cooperativism.

To apply, please submit a 1-4 page(s) position paper explaining your interests in design and cooperativism in the ACM Submission Template format by September 1, 2020 through our website https://sharingcoopnordichi2020.wordpress.com. To promote broader participation from design practitioners, and community activists, we offer the option of submitting alternative material in the form of a design portfolio, a short manifesto, or the like.

\section{RECRUITMENT AND PARTICIPATION}

Participants for the workshop will be recruited from the HCI community, attendees of previous $\mathrm{CHI}$ and CSCW workshops, and SIGs on designing for sharing and the sharing economy (e.g., [3]), and the extended research networks of the workshop organizers. Given the relevance of political economy and alternative economic models 
to the workshop's themes, we also seek participation of scholars with a background in economic sociology and anthropology. We will reach out to the international, interdisciplinary COST Action "From Sharing to Caring" (http://sharingandcaring.eu), a network of actors in and beyond academia, focused on developing sociotechnical implications of the collaborative economy. We will also circulate the call for participation on relevant listservs and through social media. Finally, we will extend invitations to design practitioners, sharing economy experts and local community protagonists in the Nordics, leveraging the location of NordiCHI 2020 to ensure the cross-discipline nature of the discussion

\section{ORGANIZERS}

Ozge Subasi is an Assistant Professor at the College of Social Sciences and Humanities at Koç University. Her research is on cocreation of new services and designs with undervalued populations, based on fieldwork in community settings, usually with crafty artifacts.

Anton Fedosov is a Postdoctoral Researcher at People and Computing Lab at the University of Zurich in Switzerland. His research interests lie at the intersection of constructive design research and non-profit approaches to collaborative economies with a particular focus on interaction and user experience design for supporting platforms and services.

Oliver Bates is a Senior Research Associate at the School of Computing and Communications at Lancaster University (UK) and member of the worker cooperative Cetis. Motivated by issues of environmental and social injustice, his research focuses on how technologies can promote environmental and social sustainability. His current work focuses on the design of technology for empowering gig economy couriers.

Airi Lampinen is an Associate Professor in Human-Computer Interaction at Stockholm University in Sweden and a Docent in Social Psychology at the University of Helsinki in Finland. She has studied interpersonal dynamics in peer-to-peer exchange extensively. Her ongoing research focuses on interpersonal challenges in sharing economies and alternative, member-driven peer-to-peer initiatives.

Ann Light is Professor of Design and Creative Technology at the University of Sussex (UK) and Professor at Malmo University (Sweden). She specializes in the social impact of technology, and particularly the deployment of platforms. Her design work concerns innovation in social process, social justice and sustainability, researched using participatory methods.

\section{ACKNOWLEDGMENTS}

This work was supported by Koç University grant no. KSF 20-15, Forschungskredit of the University of Zurich, grant no. FK-20-021, Swedish Research Council grant no. 2017-05382_3, the EPSRC grant no. EP/S027726/1, and the COST Action CA16121 From Sharing to Caring.

\section{REFERENCES}

[1] Tawanna R. Dillahunt, Jason Lam, Alex Lu, and Earnest Wheeler. 2018. Designing Future Employment Applications for Underserved Job Seekers: A Speed Dating Study. In Proceedings of the 2018 Designing Interactive Systems Conference (Hong
Kong, China) (DIS '18). Association for Computing Machinery, New York, NY, USA, 33-44. https://doi.org/10.1145/3196709.3196770

[2] Anton Fedosov, Masako Kitazaki, William Odom, and Marc Langheinrich. 2019. Sharing Economy Design Cards. In Proceedings of the 2019 CHI Conference on Human Factors in Computing Systems (Glasgow, Scotland Uk) (CHI '19). Association for Computing Machinery, New York, NY, USA, 1-14. https: //doi.org/10.1145/3290605.3300375

[3] Anton Fedosov, Airi Lampinen, Tawanna R. Dillahunt, Ann Light, and Coye Cheshire. 2019. Cooperativism and Human-Computer Interaction. In Extended Abstracts of the 2019 CHI Conference on Human Factors in Computing Systems (Glasgow, Scotland Uk) (CHI EA '19). Association for Computing Machinery, New York, NY, USA, 1-4. https://doi.org/10.1145/3290607.3311751

[4] Mareike Glöss, Moira McGregor, and Barry Brown. 2016. Designing for Labour: Uber and the On-Demand Mobile Workforce. In Proceedings of the 2016 CHI Conference on Human Factors in Computing Systems (San Jose, California, USA) (CHI '16). Association for Computing Machinery, New York, NY, USA, 1632-1643. https://doi.org/10.1145/2858036.2858476

[5] Eleni Katrini. 2018. Sharing Culture: On definitions, values, and emergence. The Sociological Review 66, 2 (2018), 425-446. https://doi.org/10.1177/ 0038026118758550

[6] Airi Lampinen and Barry Brown. 2017. Market Design for HCI: Successes and Failures of Peer-to-Peer Exchange Platforms. In Proceedings of the $2017 \mathrm{CHI}$ Conference on Human Factors in Computing Systems (Denver, Colorado, USA) (CHI '17). Association for Computing Machinery, New York, NY, USA, 4331-4343. https://doi.org/10.1145/3025453.3025515

[7] Airi Lampinen, Vilma Lehtinen, Coye Cheshire, and Emmi Suhonen. 2013. Indebtedness and Reciprocity in Local Online Exchange. In Proceedings of the 2013 Conference on Computer Supported Cooperative Work (San Antonio, Texas, USA) (CSCW'13). Association for Computing Machinery, New York, NY, USA, 661-672. https://doi.org/10.1145/2441776.2441850

[8] Ann Light. 2019. Designing the economics of the sharing economy: towards sustainable management. Edward Elgar Publishing, Cheltenham, UK. https: //www.elgaronline.com/view/edcoll/9781788110532/9781788110532.00015.xml

[9] Ann Light and Clodagh Miskelly. 2019. Platforms, scales and networks: meshing a local sustainable sharing economy. Computer Supported Cooperative Work (CSCW) 28, 3-4 (2019), 591-626.

[10] Bonnie Nardi. 2019. Design in the Age of Climate Change. She fi: The fournal of Design, Economics, and Innovation 5, 1 (2019), 5-14. 